\title{
Intravascular near-infrared fluorescence molecular imaging of atherosclerosis: toward coronary arterial visualization of biologically high-risk plaques
}

\author{
Marcella A. Calfon \\ Massachusetts General Hospital \\ Cardiovascular Research Center and Cardiology Division \\ 185 Cambridge Street \\ Simches Building Room 3206 \\ Boston, Massachusetts 02114
}

\section{Claudio Vinegoni}

Massachusetts General Hospital

Center for Systems Biology and

Center for Molecular Imaging Research

185 Cambridge Street

Boston, Massachusetts 02114

\author{
Vasilis Ntziachristos \\ Helmholtz Center Munich \\ Ingolstädter Landstraße 1 \\ D-85764 Neuherberg, Germany \\ and \\ Technical University of Munich \\ Munich, Germany
}

\section{Farouc A. Jaffer}

Massachusetts General Hospital

Cardiovascular Research Center and Cardiology Division and

Center for Molecular Imaging Research

185 Cambridge Street, Room 3206

Boston, Massachusetts 02114

\begin{abstract}
New imaging methods are urgently needed to identify highrisk atherosclerotic lesions prior to the onset of myocardial infarction, stroke, and ischemic limbs. Molecular imaging offers a new approach to visualize key biological features that characterize high-risk plaques associated with cardiovascular events. While substantial progress has been realized in clinical molecular imaging of plaques in larger arterial vessels (carotid, aorta, iliac), there remains a compelling, unmet need to develop molecular imaging strategies targeted to high-risk plaques in human coronary arteries. We present recent developments in intravascular near-IR fluorescence catheter-based strategies for in vivo detection of plaque inflammation in coronary-sized arteries. In particular, the biological, light transmission, imaging agent, and engineering principles that underlie a new intravascular near-IR fluorescence sensing method are discussed. Intravascular near-IR fluorescence catheters appear highly translatable to the cardiac catheterization laboratory, and thus may offer a new in vivo method to detect high-risk coronary plaques and to assess novel atherosclerosis biologics. $\odot 2010$ Society of Photo-Optical Instrumentation Engineers. [DOI: $10.1117 / 1.3280282]$
\end{abstract}

Keywords: atherosclerosis; imaging; fluorescence; inflammation; catheter; intravascular; molecular imaging; optical imaging.

Paper 09190SSR received May 11, 2009; revised manuscript received Jul. 27, 2009; accepted for publication Jul. 29, 2009; published online Jan. 14, 2010.

\section{Molecular Imaging of Atherosclerosis: Motivations}

Progression of asymptomatic atherosclerotic lesions is the predominant cause of myocardial infarction, stroke, and ischemic limbs. As such life-threatening events continue to occur despite advances in medical therapies, identification of high-risk lesions will be critical in preventing the occurrence of devastating cardiovascular events. Current atherosclerosis imaging strategies focus largely on the anatomic and physiologic characteristics of vessel narrowing as a consequence of vessel stenosis. While useful, these approaches provide little insight into the biology of atherosclerosis, a critical aspect of plaque progression and complications. ${ }^{1}$ Identifying the molecular or cellular determinants of high-risk (or "vulnerable") plaques therefore appears a logical approach to understanding and treating atherosclerosis. ${ }^{2}$ Toward this end, in vivo molecular imaging is a promising strategy to identify key biological

Address all correspondence to: Farouc Jaffer, MD, PhD, Massachusetts General Hospital, Cardiovascular Research Center and Cardiology Division, 185 Cambridge St., Simches Bldg. Rm. 3206, Boston, Massachusetts 02114. Tel: 617 724-9353; Fax: 617-643-3451; E-mail: fjaffer@mgh.harvard.edu processes that govern atherosclerosis, including inflammation, apoptosis, oxidative stress, and angiogenesis. 3,4

\section{Imaging of Atheroma Inflammation}

Atherosclerosis is an inflammatory disease driven by the unregulation of leukocyte adhesion molecules and subsequent recruitment of macrophages and $\mathrm{T}$ cells within the vessel wall. ${ }^{1}$ Once resident in the intima, macrophages imbibe lipid, promoting lipid-laden foam cell formation, smooth muscle proliferation, and localized and systemic inflammation typified by the production of destabilizing proteases, cytokines, and oxidants. Nascent atheromata consequently undergo expansive (positive) remodeling, and years later undergo constrictive (negative) remodeling that results in stenosis formation. During this transformative period, plaques may demonstrate high-risk features including inflammation, a large necrotic core, and a thin fibrous cap. ${ }^{2}$ Such plaques, termed high-risk or vulnerable, may become acutely disrupted, leading to thrombus formation and acute myocardial infarction.

1083-3668/2010/15(1)/011107/6/\$25.00 @ 2010 SPIE 
The inflammatory cascade has yielded a number of highsensitivity molecular imaging targets. ${ }^{3,4}$ Macrophages are the critical effectors cell in atherosclerosis and have been clinically imaged by positron emission tomography (PET), magnetic resonance imaging (MRI), and nuclear approaches. Imaging of augmented protease activities (cysteine proteases, matrix metalloproteinases) that destabilize the fibrous cap overlying plaques is a promising, translatable, near-IR fluorescence (NIRF) imaging method to detect plaque inflammation. Leukocyte adhesion molecules [e.g., vascular cell adhesion molecule (VCAM)-I] are another attractive imaging target due to their ability to report on early plaque inflammation, and have been imaged by MRI, NIRF, and ultrasound molecular imaging approaches. In addition, strategies to identify plaque apoptosis, accumulation of oxidized lipids, and angiogenesis are exciting options to identify high-risk plaques. For a detailed discussion of molecular imaging agent reporters for atherosclerosis, see several recent reviews.

\section{NIRF as a Molecular Imaging Platform}

NIRF imaging has emerged as powerful in vivo approach for molecular imaging of atherosclerosis, due to (1) its high intrinsic (picomolar) sensitivity, (2) a broad array of attachment chemistries for targeted and activatable imaging agents (discussed later in this paper), (3) efficient transmission of light in the near-IR (NIR) window, (4) a wide array of detection systems spanning ultrahigh resolution (confocal microscopy) to mesoscopic resolution (e.g., catheter-based reflectance imaging) to macroscopic resolution (e.g., fluorescence molecular tomography), and (5) substantial clinical translational capabilities from both a hardware standpoint (e.g., NIRF catheters based on clinical catheters for optical coherence tomography) and imaging agent standpoint [i.e., indocyanine green, a NIR fluorochrome that is Food and Drug Administration (FDA) approved for retinal angiography].

\subsection{Photonic Transmission in the NIR Window}

Compared to the visible light range, the NIR spectral region, and more specifically the 650 to $950-\mathrm{nm}$ window, offers attractive characteristics for optical imaging due to lower light absorption by hemoglobin in this wavelength band. This enables practical photon detection even after propagation through several centimeters in tissue, for example, through more than $10 \mathrm{~cm}$ in the human breast ${ }^{5-7}$ and more than 3 to $4 \mathrm{~cm}$ in muscle tissue or in functional brain measurements. ${ }^{8}$ By comparison, depths of only a few millimeters of tissue can be visualized with similar SNR statistics in the blue, green, or red light regions, although the exact penetration depth remains strongly dependent on the wavelength selection.

The NIR region is similarly crucial when considering intravascular applications. While the geometrical dimensions in catheter-based vessel imaging are significantly reduced compared to breast or brain imaging applications, the blood concentration within vessels is significantly higher than in breast, brain, or muscle tissue, leading to a corresponding increase in the absorption coefficient. It is therefore important to utilize NIR wavelengths to achieve high sensitivity through whole blood. In addition, tissue exhibits reduced autofluorescence in the NIR compared to the visible light range. ${ }^{9}$ This advantage augments the contrast available to imaging and reduces the need for multispectral methods that may otherwise be necessary to decompose the effects of autofluorescence from the signal detected from a reporter fluorochrome.

\subsection{Protease-Activatable NIRF Imaging Agents}

The chemical design of a new class of activatable imaging agents based on protease-mediated cleavage of quenched substrates has dramatically improved the sensitivity of in vivo NIRF imaging. Protease-activatable probes are comprised of 15 to 20 NIR fluorochromes that are conjugated to a high molecular weight $(\sim 500 \mathrm{kD})$ methyl poly-(ethylene glycol) (MPEG) poly-L-lysine backbone. A radio labeled version of this backbone safely completed clinical trials. ${ }^{10}$ As the fluorochromes exist in close proximity, self-quenching of the molecule occurs, leading to an optically "silent" imaging agent. As opposed to conventional fluorochromes (or radioisotopes, magnetic reporters, or microbubbles), these quenched fluorescence substrates generate minimal background signal after injection into the body. In a suitable protease-rich environment, cleavage of the quenched substrate occurs, liberating fluorescence. As opposed to imaging agents that report on protease presence with active site-binders, ${ }^{11,12}$ the background signal of the uncleaved NIRF activatable agent is minimal due to its engineered autoquenched design, and high target-tobackground ratios are achievable in vivo. ${ }^{13}$

In particular, in vivo imaging of inflammatory plaques using activatable NIRF probes targeted to cysteine and matrix metalloproteinases (MMPs) have yielded promising results by both intravital fluorescence microscopy (IVFM) and fluorescence molecular tomography (FMT) detection. ${ }^{14-16}$ A firstgeneration activatable NIRF agent targeted to multiple cathepsins $^{13,14}$ (cathepsin B, L, S) is now under development for clinical trials for both vascular and cancer applications. ${ }^{3}$ By interspersing an oligopeptide construct into the MPEG backbone, second-generation NIRF activatable agents can be constructed with specificities for gelatinase activity ${ }^{15}$ (MMP-2, MMP-9) and cathepsin K. ${ }^{16}$ In addition, other protease activities have also been imaged using secondgeneration NIRF activatable reporters such as thrombin, ${ }^{17}$ cathepsin $\mathrm{D},{ }^{18}$ urokinase-type plasminogen activator, ${ }^{19}$ and cathepsin $\mathrm{S}^{20}$

\subsection{Intravascular NIRF Catheter Sensing of Inflammation in Coronary-Sized Vessels}

A recently tested NIRF catheter offers a new invasive molecular imaging approach to visualize atheroma inflammation in vivo through blood in coronary-sized vessels. ${ }^{21,22}$

\subsection{NIRF Catheter Design}

The first-generation coronary artery compatible guidewire is based $^{22}$ on a clinically translatable optical coherence tomography (OCT) platform (Fig. 1). The catheter utilizes as an excitation source a laser diode at a $750 \mathrm{~nm}$ (B\&W TEK, Newark, New Jersey) and it collects fluorescence signal at $780 \mathrm{~nm}$. As the excitation and the emission channels are spectrally close to each other, the laser light is first filtered with a narrow-bandpass interference filter centered at $752 \mathrm{~nm}$ and with a 5-nm full width at half maximum (FWHM) to remove any residual laser emission present in the fluorescence detec- 
A.

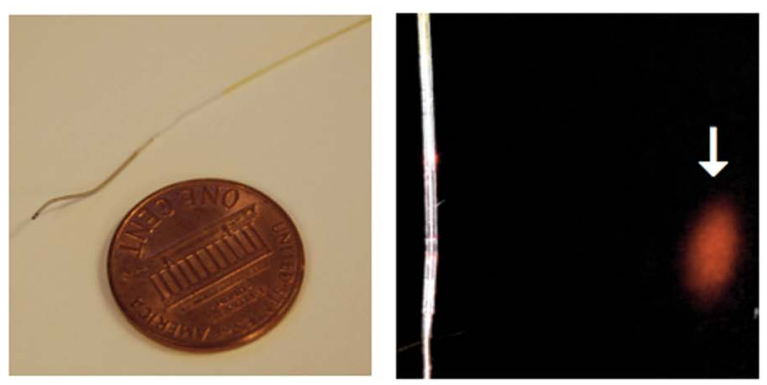

C.

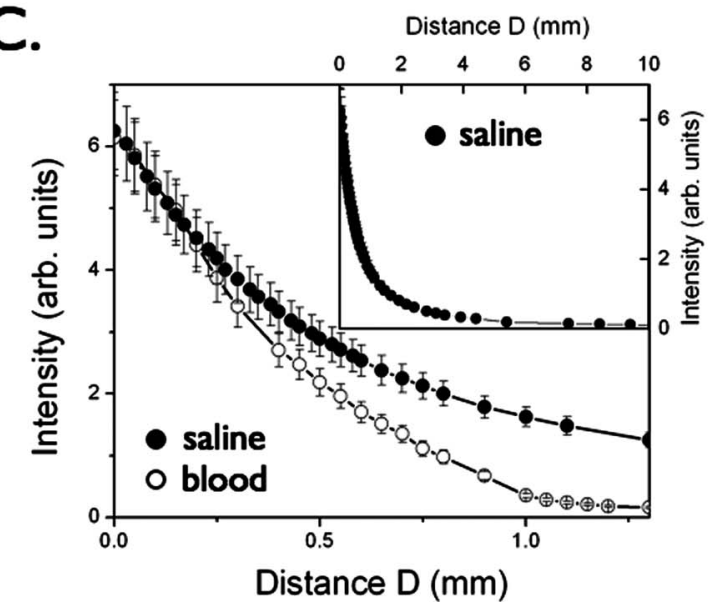

B.

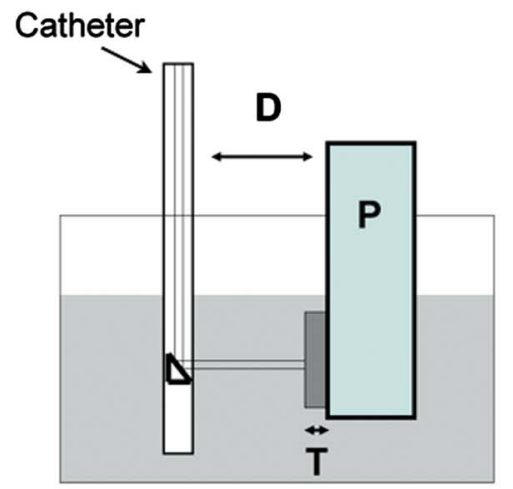

D.

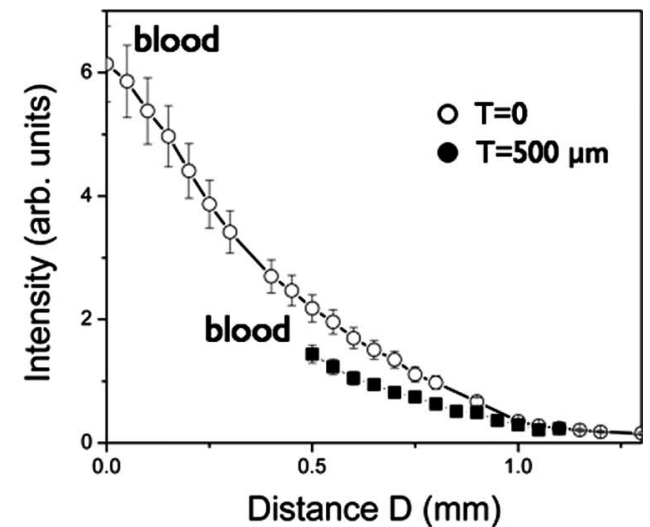

Fig. 1 Catheter prototype for intravascular sensing of NIR fluorescence signals. (a) The NIRF catheter consists of a 0.36-mm/0.014-in. floppy radiopaque tip with a maximum outer diameter of $0.48 \mathrm{~mm} / 0.019 \mathrm{in}$.. The arrow highlights the focal spot (40 $\pm 15 \mu \mathrm{m})$ for the $90-$ deg arc-sensing catheter at a distance of $2 \pm 1 \mathrm{~mm}$ (arrow). (b) Phantom experiment to measure NIR light attenuation in the presence of whole blood. Plaque $(P)$ consists of $1 \%$ Intralipid plus India ink 50 ppm plus AF750 (an NIR fluorochrome, concentration $300 \mathrm{nmol} / \mathrm{L}$ ); tissue ( $T$ : fibrous cap) consists of polyester casting resin plus titanium dioxide plus India ink; a container (gray shaded area) was filled with fresh rabbit blood or saline. The catheter was immersed in fresh rabbit blood and positioned at variable distance $(D)$ from a fluorescent phantom representing the plaque $(P)$. To mimic the presence of a fibrous cap, a solid tissue phantom of thickness $T$ was interposed between the plaque and the lumen. (c) Plot of detected NIRF signal as a function of distance $D$ in presence of blood compared to saline, showing only modest attenuation by blood. Inset, fluorescence signal decay in saline at distance of up to $10 \mathrm{~mm}$. (d) Plot of the detected NIRF signal in blood in the presence of a tissue phantom ( $T$ ) of thickness $500 \mu \mathrm{m}$ shows modest NIRF signal attenuation $(<35 \%)$ vs the case in (c) where $T=0$. Reproduced by permission from Ref. 22.

tion channel. Excitation light is then coupled into a system delivery fiber after passing through a $3-\mathrm{dB}$ beamsplitter and coupled into the dedicated catheter based on an optical coherence tomography wire (LightLab Imaging Inc., Westford, Massachusetts). The catheter is comprised of a $0.36-\mathrm{mm} / 0.014-$ in. radiopaque floppy tip that allows for $\mathrm{x}$-ray detection, and a $0.48-\mathrm{mm} / 0.019$-in. outer diameter housing containing a $62.5 / 125-\mu \mathrm{m}$ multimode fiber for maximal light collection.

A prism at the end of the catheter directs the light at 90 deg with respect to the catheter axis and focuses the light on a focal spot size of around $40 \mu \mathrm{m}$ diameter at a working distance of $2 \mathrm{~mm}$. The fluorescent light emitted by the contrast agent present within the plaque is then collected back into the catheter, guided to the beamsplitter, and coupled into a separate multimode fiber. The fluorescence signal is next filtered with a dielectrically coated dichroic filter with a cut-on wavelength of $780 \mathrm{~nm}$ to remove any further residual excitation laser component. A sensitive photomultiplier tube
(H5783-20, Hamamatsu, Shizuoka, Japan) then detects the residual filtered light.

\section{Phantom Simulations of Blood and Tissue Attenuation of NIR Light}

The effects of blood and tissue on NIRF signal attenuation were tested using a home-built atherosclerotic vessel wall phantom with fresh rabbit blood, simulated tissue, and a NIRF plaque (Fig. 1). The plaque consisted of a cuvette filled with a solution of $1 \%$ Intralipid (Liposyn, Hospira Inc., Lake Forest, Illinois) and $50 \mathrm{ppm}$ India ink (Faber-Castell, Cleveland, Ohio) to match the optical parameters of tissue ( $\mu_{a}=0.4 \mathrm{~cm}^{-1}$ and $\mu_{s}=12 \mathrm{~cm}^{-1}$, respectively). The cuvette mimicking the plaque was rendered fluorescent with an NIR fluorochrome (AF750, $300 \mathrm{nM}$, Molecular Probes, Eugene, Oregon), while the overlying nonfluorescent solid tissue phantom consisted of a polyester casting resin (Castin'Craft, ETI, Fields Landing, California) mixed with $\mathrm{TiO}_{2}$ (Sigma-Aldrich 

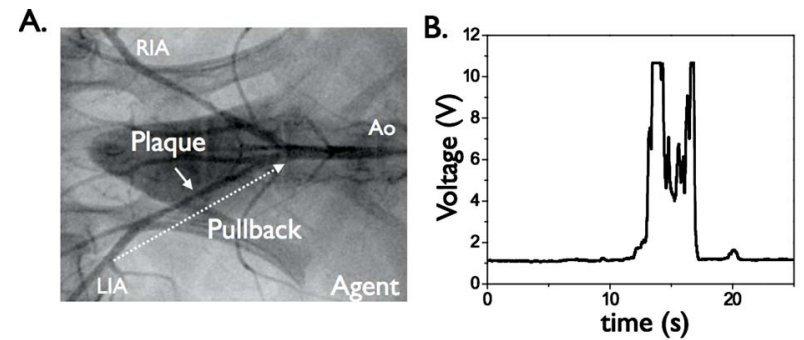

C.

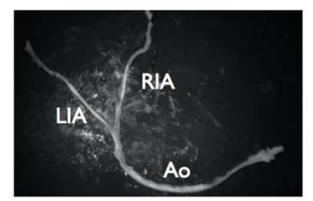

D.

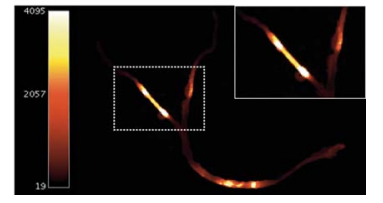

Fig. 2 Real-time, in vivo fluorescence sensing of inflammation in atherosclerotic vessels through blood: (a) repeated real-time manual pullback of the catheter performed in each iliac artery over $20 \mathrm{~s}$ (dotted arrow); (b) in rabbits that received a first-generation proteaseactivatable NIRF agent $24 \mathrm{~h}$ beforehand, strong NIRF signal was detected on pullback in iliac artery lesions [target-to-background ratio (TBR)] average of $6.8 \pm 1.9$ (vs $1.3 \pm 0.3$ in saline-injected control animals, $p<0.05$ ) and (c) and (d) ex vivo paired white light and NIRF images of atherosclerotic arteries. Augmented NIRF signal was evident in plaques from rabbits injected with the cysteine protease activatable agent. Minimal autofluorescence was noted in saline-injected control animals (data not shown). RIA, right iliac artery; LIA, left iliac artery; Ao, aorta. Modified by permission from Ref. 22.

Chemical Co. Inc., Milwaukee, Wisconsin) and India ink. The imaging catheter was next immersed in the vessel phantom (a large container filled with rabbit blood) at variable distances from the NIR fluorescent plaque.

As expected, NIR fluorescence light attenuated as function of increasing distance. However, notably, the effects of blood compared to saline were modest in the NIR with decay distance $(1 / e)$ of $500 \mu \mathrm{m}$ compared with a decay distance of $700 \mu \mathrm{m}$ in saline (Fig. 1), affirming the favorable photonic transmission properties of the NIR window. The tissue $(T)$ thickness, reflecting a simulated fibrous cap overlying the fluorescent plaque, also produced only modest effects on the NIRF signal at a 500- $\mu \mathrm{m}$ thickness. These experiments demonstrated the feasibility of NIR light detection through intravascular volumes of blood relevant for atherosclerotic plaques.

\subsection{Real-Time Intravascular Sensing of NIRF Signals Emanating from Inflamed Plaques}

The first in vivo application of the NIRF catheter was demonstrated using atherosclerotic rabbits harboring inflamed atheromata. ${ }^{22}$ Angiographically visible lesions were produced by balloon injury of the iliac arteries followed by hypercholesterolemia for 8 weeks (Fig. 2). Twenty-four hours prior to in vivo experiments, rabbits received an injection of a firstgeneration cysteine protease-activatable NIRF agent (Prosense750, VisEn Medical). The NIRF catheter was then advanced percutaneously into the iliac arteries under x-ray fluoroscopic guidance. Manual, repeated digitized pullbacks and voltage recordings were obtained through blood, without balloon occlusion or saline flushing (Fig. 2). In vivo TBRs were calculated in both normal and diseased segments. NIRF
A.

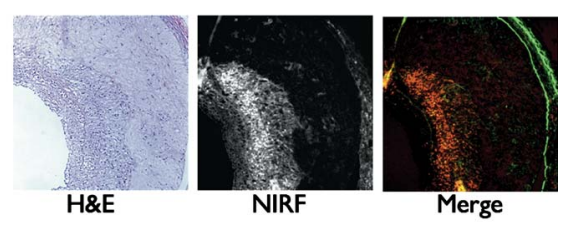

B.

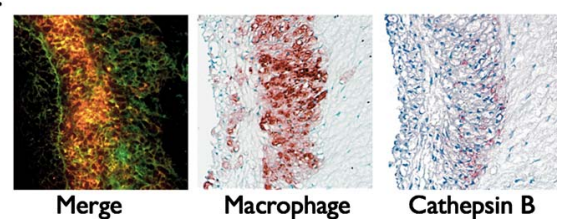

Fig. 3 Correlative histopathology and fluorescence microscopy of atheroma sections of a rabbit injected with the cathepsin-activatable NIRF agent and following intravascular NIRF sensing. (a) left, hematoxylin and eosin (H\&E); middle, NIRF microscopy (pseudocolored red); right, merged NIRF and 500-nm autofluorescence (pseudocolored green) image. Images acquired at $\times 100$ magnification. (b) Left, abundant NIRF signal from activation of the protease-activatable agent (red) overlying diffuse autofluorescence (green); middle and right, immunoreactive macrophages and cathepsin $B$ respectively colocalize with the NIRF signal. Images acquired at $\times 200$ magnification. Modified by permission from Ref. 22. (Color online only.)

signal profiles readily distinguished atheroma from normal segments, as well as from atheroma of saline-injected control rabbits. High in vivo plaque TBRs were detected with values of $6.8 \pm 1.9$, compared to saline controls of $1.3 \pm 0.3$ $(p<0.05)$. To confirm that augmented voltage was plaque specific, the catheter was readvanced to lesions visible on the angiogram. The static NIRF signal was recorded adjacent to plaques and to normal-appearing vessel wall areas on angiography.

To further assess the effect of blood absorption on the detected NIRF signal, vessel occlusion and saline flushing $(3 \mathrm{ml}$ over $4 \mathrm{~s}$ ) were performed through a balloon wedge catheter. In the protease agent group only, differences in flush profiles were evident between plaque areas and normal segments. In plaque areas, saline flushing augmented the detected NIRF signal with a peak signal increase of up to $74 \pm 18 \%$. In the protease agent group, macroscopic fluorescence reflectance imaging (FRI) revealed NIRF signal in plaques but not in the uninjured, normal-appearing vessel segment. The in vivo and ex vivo peak plaque TBRs correlated well $(r=0.82, p<0.01)$, demonstrating reasonable in vivo quantification methodology. In vitro histopathology confirmed colocalization of NIRF signal with cathepsin B and macrophages within atheroma (Fig. 3 ), and demonstrated reduced plaque autofluorescence in the NIR range compared to the visible range, as expected. ${ }^{22}$ This study thus provided the first demonstration of in vivo molecular imaging of plaque inflammation in coronary-sized vessels. Due to the ability to obtain high TBRs in vivo through blood in real time, this approach appears promising for rapid coronary artery screening in vivo. Additional studies will clarify the attenuation effects of blood in larger vessels (e.g., $3.5 \mathrm{~mm}$ diameter) than tested in this study (2.25 to $2.5 \mathrm{~mm}$ diameter).

\subsection{Intravascular NIRF Catheter Design: Future Directions}

While original intravascular fluorescence imaging designs ${ }^{21,22}$ have demonstrated feasibility based on 1-D signal acquisition, 
it is crucial to develop catheter systems with the ability to provide 3-D imaging of vessel segments as necessary for comprehensive coronary arterial imaging. Of importance are methodologies that can offer accurate visualization and impart quantification within the challenging imaging conditions of a blood vessel. A preferred mode of operation is the use of an enclosed rotating fiber, similar to those utilized in intravascular optical coherence tomography systems. ${ }^{23}$ The combination of the rotating fiber with a pullback mechanism further enables the longitudinal visualization of vessels. Typically, the selection of a large fiber diameter offers high collection efficiency in multimode propagation. A crucial design parameter is the point spread function of the illumination and detection profile, in particular, the beam waist size of the focal spot and the Rayleigh length that define the metrics of energy deposition confinement on the vessel as a function of vessel diameter. Extended Rayleigh lengths (i.e., the length along which the beam stays focused) is particularly important for maintaining relative uniformity of illumination (energy deposition) and resolution during the imaging operation.

The design of rotating NIRF imaging catheters enables the possibility of seamless operation with other vessel imaging modalities, for example, with intravascular OCT, intravascular optical frequency domain imaging, ${ }^{24}$ intravascular NIR spectroscopy, ${ }^{25}$ or intravascular ultrasound. While chromatic aberrations of the focusing optics must be considered when markedly different spectral zones are utilized for OCT versus fluorescence imaging, the relatively relaxed optical conditions for the noncoherent fluorescence detection will allow for design practices common in currently available intravascular OCT systems. Another advance in intravascular NIRF imaging possible is to employ molecular optoacoustic imaging for visualizing common fluorochromes and other chromophoric reporter molecules, ${ }^{26,27}$ or tissue composition features such as fibrous and lipid components. The use of optoacoustics has the capacity to further resolve depth, reaching resolution and penetration capacity that are common to ultrasound systems, but imparting new contrast mechanisms for atherosclerosis differentiation.

\section{Conclusions}

The development of a coronary artery-compatible NIRF sensing catheter coupled with a high-sensitivity proteaseactivatable agent has enabled the real-time sensing of plaque inflammation through blood. Anticipated development of second-generation (imaging, multispectral, optoacoustic) and third-generation (multimodality) NIRF catheters are poised to enable integrated molecular and architectural assessment of coronary plaques in the cardiac catheterization laboratory. The availability of clinical coronary NIRF catheters with FDAapproved NIRF imaging agents will enable (1) new insights into atherosclerosis biology in living patients (in particular, the temporal and spatial characteristics of inflammation in coronary arteries), (2) understanding an individual plaque's risk of inducing myocardial infarction based on its underlying biological profile, and (3) assessment of novel biologics designed to attenuate inflammation in coronary atherosclerotic plaques.

\section{Disclosures}

V.N. and F.A.J. hold equity in VisEn Medical, Bedford, Massachusetts.

\section{Acknowledgments}

Support for this work was provided by the Howard Hughes Medical Institute Career Development Award (FAJ), the American Heart Association Scientist Development Grant (FAJ), the Donald W. Reynolds Foundation (FAJ), and the MGH William Schreyer Fellowship (MAC).

\section{References}

1. G. K. Hansson, "Inflammation, atherosclerosis, and coronary artery disease," N. Engl. J. Med. 352, 1685-1695 (2005).

2. M. Naghavi, P. Libby, E. Falk, S. W. Casscells, S. Litovsky, J. Rumberger, J. J. Badimon, C. Stefanadis, P. Moreno, G. Pasterkamp, Z. Fayad, P. H. Stone, S. Waxman, P. Raggi, M. Madjid, A. Zarrabi, A. Burke, C. Yuan, P. J. Fitzgerald, D. S. Siscovick, C. L. de Korte, M. Aikawa, K. E. Juhani Airaksinen, G. Assmann, C. R. Becker, J. H. Chesebro, A. Farb, Z. S. Galis, C. Jackson, I. K. Jang, W. Koenig, R. A. Lodder, K. March, J. Demirovic, M. Navab, S. G. Priori, M. D. Rekhter, R. Bahr, S. M. Grundy, R. Mehran, A. Colombo, E. Boerwinkle, C. Ballantyne, W. Insull, Jr., R. S. Schwartz, R. Vogel, P. W. Serruys, G. K. Hansson, D. P. Faxon, S. Kaul, H. Drexler, P. Greenland, J. E. Muller, R. Virmani, P. M. Ridker, D. P. Zipes, P. K. Shah, and J. T. Willerson, "From vulnerable plaque to vulnerable patient: a call for new definitions and risk assessment strategies: part I," Circulation 108, 1664-1672 (2003).

3. F. A. Jaffer, P. Libby, and R. Weissleder, "Molecular imaging of cardiovascular disease," Circulation 116, 1052-1061 (2007).

4. J. Sanz and Z. A. Fayad, "Imaging of atherosclerotic cardiovascular disease," Nature (London) 451, 953-957 (2008).

5. B. W. Pogue, S. P. Poplack, T. O. McBride, W. A. Wells, K. S. Osterman, U. L. Osterberg, and K. D. Paulsen, "Quantitative hemoglobin tomography with diffuse near-infrared spectroscopy: pilot results in the breast," Radiology 218, 261-266 (2001).

6. V. Ntziachristos, A. G. Yodh, M. Schnall, and B. Chance, "Concurrent MRI and diffuse optical tomography of breast after indocyanine green enhancement," Proc. Natl. Acad. Sci. U.S.A. 97, 2767-2772 (2000).

7. Q. Fang, S. A. Carp, J. Selb, G. Boverman, Q. Zhang, D. B. Kopans, R. H. Moore, E. L. Miller, D. H. Brooks, and D. A. Boas, "Combined optical imaging and mammography of the healthy breast: optical contrast derived from breast structure and compression," IEEE Trans. Med. Imaging 28, 30-42 (2009).

8. R. D. Hoge, M. A. Franceschini, R. J. Covolan, T. Huppert, J. B. Mandeville, and D. A. Boas, "Simultaneous recording of taskinduced changes in blood oxygenation, volume, and flow using diffuse optical imaging and arterial spin-labeling MRI," Neuroimage 25, 701-707 (2005)

9. M. G. Muller, I. Georgakoudi, Q. Zhang, J. Wu, and M. S. Feld, "Intrinsic fluorescence spectroscopy in turbid media: disentangling effects of scattering and absorption," Appl. Opt. 40, 4633-4646 (2001).

10. R. J. Callahan, A. Bogdanov, Jr., A. J. Fischman, T. J. Brady, and R. Weissleder, "Preclinical evaluation and phase I clinical trial of a 99mTc-labeled synthetic polymer used in blood pool imaging," AJR, Am. J. Roentgenol. 171, 137-143 (1998).

11. M. Schafers, B. Riemann, K. Kopka, H. J. Breyholz, S. Wagner, K. P. Schafers, M. P. Law, O. Schober, and B. Levkau, "Scintigraphic imaging of matrix metalloproteinase activity in the arterial wall in vivo," Circulation 109, 2554-2559 (2004).

12. E. Lancelot, V. Amirbekian, I. Brigger, J. S. Raynaud, S. Ballet, C. David, O. Rousseaux, S. Le Greneur, M. Port, H. R. Lijnen, P. Bruneval, J. B. Michel, T. Ouimet, B. Roques, S. Amirbekian, F. Hyafil, E. Vucic, J. G. Aguinaldo, C. Corot, and Z. A. Fayad, "Evaluation of matrix metalloproteinases in atherosclerosis using a novel noninvasive imaging approach," Arterioscler., Thromb., Vasc. Biol. 28, 425-432 (2008).

13. R. Weissleder, C. H. Tung, U. Mahmood, and A. Bogdanov, Jr., "In vivo imaging of tumors with protease-activated near-infrared fluorescent probes," Nat. Biotechnol. 17, 375-378 (1999). 
14. J. Chen, C. H. Tung, U. Mahmood, V. Ntziachristos, R. Gyurko, M. C. Fishman, P. L. Huang, and R. Weissleder, "In vivo imaging of proteolytic activity in atherosclerosis," Circulation 105, 2766-2771 (2002).

15. J. O. Deguchi, M. Aikawa, C. H. Tung, E. Aikawa, D. E. Kim, V. Ntziachristos, R. Weissleder, and P. Libby, "Inflammation in atherosclerosis: visualizing matrix metalloproteinase action in macrophages in vivo," Circulation 114, 55-62 (2006).

16. F. A. Jaffer, D. E. Kim, L. Quinti, C. H. Tung, E. Aikawa, A. N. Pande, R. H. Kohler, G. P. Shi, P. Libby, and R. Weissleder, "Optical visualization of cathepsin $\mathrm{K}$ activity in atherosclerosis with a novel, protease-activatable fluorescence sensor," Circulation 115, 22922298 (2007).

17. F. A. Jaffer, C. H. Tung, R. E. Gerszten, and R. Weissleder, "In vivo imaging of thrombin activity in experimental thrombi with thrombinsensitive near-infrared molecular probe," Arterioscler., Thromb., Vasc. Biol. 22, 1929-1935 (2002).

18. C. H. Tung, U. Mahmood, S. Bredow, and R. Weissleder, "In vivo imaging of proteolytic enzyme activity using a novel molecular reporter," Cancer Res. 60, 4953-4958 (2000).

19. J. K. Hsiao, B. Law, R. Weissleder, and C. H. Tung, "In vivo imaging of tumor associated urokinase-type plasminogen activator activity," J. Biomed. Opt. 11, 34013 (2006).

20. A. K. Galande, S. A. Hilderbrand, R. Weissleder, and C. H. Tung, "Enzyme-targeted fluorescent imaging probes on a multiple antigenic peptide core," J. Med. Chem. 49, 4715-4720 (2006).

21. B. Zhu, F. A. Jaffer, V. Ntziachristos, and R. Weissleder, "Develop- ment of a near infrared fluorescence catheter: operating characterisitics and feasibility for atherosclerotic plaque detection," J. Phys. D: Appl. Phys. 38, 2701-2707 (2005).

22. F. A. Jaffer, C. Vinegoni, M. C. John, E. Aikawa, H. K. Gold, A. V. Finn, V. Ntziachristos, P. Libby, and R. Weissleder, "Real-time catheter molecular sensing of inflammation in proteolytically active atherosclerosis," Circulation 118, 1802-1809 (2008).

23. G. J. Tearney, I. K. Jang, and B. E. Bouma, "Optical coherence tomography for imaging the vulnerable plaque," J. Biomed. Opt. 11, 021002 (2006).

24. S. H. Yun, G. J. Tearney, B. J. Vakoc, M. Shishkov, W. Y. Oh, A. E. Desjardins, M. J. Suter, R. C. Chan, J. A. Evans, I. K. Jang, N. S. Nishioka, J. F. de Boer, and B. E. Bouma, "Comprehensive volumetric optical microscopy in vivo," Nat. Med. 12, 1429-1433 (2006).

25. C. M. Gardner, H. Tan, E. L. Hull, J. B. Lisauskas, S. T. Sum, T. M Meese, C. Jiang, S. P. Madden, J. D. Caplan, A. P. Burke, R. Virmani, J. Goldstein, and J. E. Muller, "Detection of lipid core coronary plaques in autopsy specimens with a novel catheter-based nearinfrared spectroscopy system," JACC Cardiovasc. Imaging 1, 638648 (2008).

26. D. Razansky, C. Vinegoni, and V. Ntziachristos, "Multispectral photoacoustic imaging of fluorochromes in small animals," Opt. Lett. 32, 2891-2893 (2007).

27. D. Razansky, C. Vinegoni, and V. Ntziachristos, "Imaging of mesoscopic-scale organisms using selective-plane optoacoustic tomography," Phys. Med. Biol. 54, 2769-2777 (2009). 